\title{
LINC00210 exerts oncogenic roles in glioma by sponging miR-328
}

\author{
ZHIFEI WANG, HAO WU, HUI YAN, TAO CAI, JIN DAI and QIANG LIU \\ Department of Neurosurgery, Third Xiangya Hospital of Central South University, Changsha, Hunan 410013, P.R. China
}

Received March 9, 2019; Accepted August 21, 2020

DOI: $10.3892 / \mathrm{etm} .2020 .9266$

\begin{abstract}
Long non-coding RNAs (lncRNAs) have been reported to serve key roles in human cancer types, including glioma. However, to the best of our knowledge, the expression and function of IncRNA LINC00210 in glioma have not previously been investigated. The present study was conducted to explore the regulatory role of LINC00210 in glioma cells. The present study demonstrated that LINC00210 was significantly upregulated in glioma tissues, and high expression of LINC00210 was significantly associated with advanced clinical stage and poor prognosis in patients with glioma. It was found that LINC00210 knockdown significantly inhibited the proliferation and migration of U251 and T98G cells. The results of luciferase reporter assays indicated that LINC00210 could directly target microRNA (miR)-328 in glioma cells, and miR-328 expression was negatively correlated with LINC00210 expression in glioma tissues. LINC00210 knockdown significantly promoted the expression of miR-328 in U251 and T98G cells. Moreover, silencing miR-328 impaired the inhibitory effects of LINC00210 knockdown on the proliferation and migration of U251 and T98G cells. Therefore, the present results suggested that LINC00210 may exert an oncogenic role in glioma via sponging miR-328.
\end{abstract}

\section{Introduction}

Glioma is the most common malignant tumor in the brain and has a very poor prognosis as most patients with glioma are diagnosed at advanced stages $(1,2)$. While increased effort has been made to investigate the etiology of glioma in recent decades, the underlying molecular mechanisms remain poorly understood $(3,4)$. Therefore, identifying these molecular mechanisms is of great importance for developing novel strategies for the diagnosis and treatment of glioma (3-5).

Long non-coding RNAs (lncRNAs), a type of single strand non-coding RNA > 200 nucleotides in length, have been

Correspondence to: Dr Qiang Liu, Department of Neurosurgery, Third Xiangya Hospital of Central South University, 138 Tongzipo Road, Changsha, Hunan 410013, P.R. China

E-mail: liuqiang19780303@163.com

Key words: glioma, LINC00210, microRNA-328, proliferation, migration reported to serve key roles in various biological processes, such as cell proliferation, migration, apoptosis and tumorigenesis $(6,7)$. For instance, the lncRNA steroid receptor RNA activator promotes vascular smooth muscle cell proliferation and neointimal hyperplasia via the MEK/ERK/cAMP response element-binding protein pathway (8). It has also been shown that IncRNA H19 regulates trophoblastic spheroid adhesion (9). Moreover, a large number of lncRNAs have been observed to be deregulated during the development and progression of human cancer types, including glioma, and some have been suggested as promising therapeutic targets $(3,7,10)$. For example, upregulation of IncRNA AFAP1-AS1 predicts poor prognosis in patients with cervical cancer, as well as promotes the migration and invasion of cervical cancer cells (11). Jiang et al (12) also reported that silencing the lncRNA HOXA distal transcript antisense RNA suppressed prostate cancer cell proliferation and enhanced cell sensitivity to cisplatin by inhibiting the Wnt/ $\beta$-catenin pathway.

MicroRNAs (miRNAs/miRs), a type of small non-coding RNA with $\sim 20$ nucleotides, are regulators of gene expression and are involved in a variety of physiological and pathological processes, such as differentiation, development, cell proliferation and motility and tumorigenesis (13). IncRNAs can competitively bind miRNAs and negatively affect miRNA expression levels $(13,14)$. For instance, lncRNA ANRIL inhibits the senescence of vascular smooth muscle cells via regulating miR-181a (15), while IncRNA LINC01234 promotes the proliferation of colon cancer cells by regulating the expression of miR-642a-5p (16).

LINC00210, a newly discovered IncRNA, can drive Wnt/ $\beta$-catenin signaling activation and thus liver tumor progression (17). However, to the best of our knowledge, no previous study has focused on the expression and function of LINC00210 in glioma. Therefore, the present study aimed to evaluate the clinical significance of LINC00210 expression in glioma. In addition, the role of LINC00210 in the regulation of glioma cell proliferation and migration, as well as the potential molecular mechanism were examined.

\section{Materials and methods}

Clinical tissue samples. The current study collected 54 glioma tissues and 14 healthy brain tissues (with $1 \mathrm{~cm}$ distance from the glioma tissues) from 54 patients with primary glioma at The Third Xiangya Hospital of Central South University (Changsha, China) between March 2011 and June 2013. These patients with glioma included 33 men and 21 women who were 
32-66 years old, with a mean age of 48.6 years. No patient with glioma received adjuvant treatment before surgery. The tissues were stored at $-80^{\circ} \mathrm{C}$ until use. These patients were followed-up for 5 years for survival analysis. This study was approved by the Ethics Committee of The Third Xiangya Hospital of Central South University, and informed written consent was obtained from these patients with glioma.

Cell culture and transfection. Human glioma cell lines, including U251, T98G, U-373MG Uppsala and U-87MG, were obtained from the Cell Bank of the Chinese Academy of Sciences, and normal human astrocytes (NHAs) were purchased from Lonza Group, Ltd. The U-87MG cell line is the original glioblastoma cell line established in the University of Uppsala. The authentication of these cell lines has been confirmed via STR profiling. Cells were cultured in DMEM (Thermo Fisher Scientific, Inc.) supplemented with 10\% FBS (Thermo Fisher Scientific, Inc.) at $37^{\circ} \mathrm{C}$ with $5 \% \mathrm{CO}_{2}$.

For cell transfection, Lipofectamine ${ }^{\circledR} 2000$ (Thermo Fisher Scientific, Inc.) was used to transfect U251 and T98G cells with $100 \mathrm{nM}$ negative control (NC) small interfering (si)RNA (cat. no. AM4611), LINC00210 siRNA1 (cat. no. n545578), LINC00210 siRNA2 (cat. no. n545579), miR-NC (cat. no. 4464058), miR-328 mimic (cat. no. 4464066) or miR-328 inhibitor (cat. no. 4464084; anti-miR-328 group), according to the manufacturer's instructions. All of these transfects were obtained from Thermo Fisher Scientific, Inc. At $48 \mathrm{~h}$ after cell transfection, reverse transcription-quantitative PCR (RT-qPCR) was performed.

$R T$ - $q P C R$. Total RNA was extracted from clinical tissue samples and cell lines using TRIzol ${ }^{\circledR}$ reagent (Thermo Fisher Scientific, Inc.). RNA was then reverse transcribed into cDNA using High Capacity cDNA Reverse Transcription kit (cat. no. 4368814; Thermo Fisher Scientific, Inc.) according to the manufacturer's instructions. The reverse transcription was performed as follows: $16^{\circ} \mathrm{C}$ for $30 \mathrm{~min}$, followed by $42^{\circ} \mathrm{C}$ for $30 \mathrm{~min}$ and $85^{\circ} \mathrm{C}$ for $5 \mathrm{~min}$. Then, cDNA was used to conduct qPCR using a miScript SYBR ${ }^{\circledR}$ Green PCR kit (Qiagen GmbH) on an ABI 7500 PCR machine (Thermo Fisher Scientific, Inc.). The PCR conditions were as follows: Initial denaturation at $95^{\circ} \mathrm{C}$ for $3 \mathrm{~min}$, followed by 40 cycles of $95^{\circ} \mathrm{C}$ for $15 \mathrm{sec}$, $60^{\circ} \mathrm{C}$ for $15 \mathrm{sec}$ and $72^{\circ} \mathrm{C}$ for $15 \mathrm{sec}$, and final extension at $72^{\circ} \mathrm{C}$ for $10 \mathrm{~min}$. U6 was used as the internal reference for miR-328, while GAPDH was used as the internal reference for LINC00210. The $2^{-\Delta \Delta C q}$ method was used for analyzing the expression (18). The primer sequences were as follows: LINC00210 forward, 5'-AACACGTTAGCGGGTTCTCA-3' and reverse, 5'-TCAAAAACCACCGAGGGAGG-3'; GAPDH forward, 5'-CTGGGCTACACTGAGCACC-3' and reverse, 5'-AAGTGGTCGTTGAGGGCAATG-3'; miR-328 forward, 5'-AACGAGACGACGACAGAC-3' and reverse, 5'-GGGGGG GCAGGAGGGGCTCAGGG-3'; and U6 forward, 5'-CTC GCTTCGGCAGCACA-3' and reverse, 5'-AACGCTTCACGA ATTTGCGT-3'.

Cell Counting Kit (CCK)-8 assays. For cell proliferation analysis, the transfected $\mathrm{U} 251$ and $\mathrm{T} 98 \mathrm{G}$ cells $(5,000$ cells/well) were seeded in a $96-$ well plate and cultured at $37^{\circ} \mathrm{C}$ for $0,24,48$ or $72 \mathrm{~h}$, as indicated. Then, according to the manufacturer's instructions, $10 \mu \mathrm{lCCK}-8$ solution (Beyotime Institute of Biotechnology) was added and the cells were incubated at $37^{\circ} \mathrm{C}$ for $1 \mathrm{~h}$. Next, the absorbance was determined at a wavelength of $450 \mathrm{~nm}$ with a microplate reader (Bio-Rad Laboratories, Inc.).

Wound healing assays. Transfected U251 and T98G cells ( $2 \times 10^{5}$ cells/well) were seeded in a 24 -well plate and cultured at $37^{\circ} \mathrm{C}$ to $90 \%$ confluence. A sterilized $200-\mu 1$ pipette tip was then used to scratch the cells to generate a line. Cells were washed twice with DPBS (Thermo Fisher Scientific, Inc.), serum-free DMEM was added and images were captured under a light microscope (magnification, x40; DMI 4000B; Leica Microsystems $\mathrm{GmbH}$ ). Next, the cells were incubated at $37^{\circ} \mathrm{C}$ for another $24 \mathrm{~h}$ and imaged under a light microscope. The wounds were analyzed using ImageJ software version 1.48 (National Institutes of Health).

Luciferase reporter gene assays. The potential miR targets of LINC00210 were predicted using the bioinformatics software miRcode version 11 (http://www.mircode.org/). Dual-luciferase Target Vectors (Promega Corporation) containing wild-type (WT) and mutant type (MT) LINC00210 binding sites with miR-328 were generated. Lipofectamine 2000 was used to co-transfect U251 and T98G cells with $100 \mathrm{nM}$ miR-NC or miR-328 mimics and $100 \mathrm{ng}$ WT-LINC00210 or MT-LINC00210 luciferase reporter plasmid. After transfection for $24 \mathrm{~h}$, a Dual-Luciferase Reporter assay system kit (Promega Corporation) was used to examine the luciferase activity according to the manufacturer's instructions. The ratio of firefly to Renilla luciferase activity was determined.

Statistical analysis. The experiments were repeated three times. Data are presented as the mean \pm SD and were analyzed using SPSS 21.0 software (IBM Corp.). A two-tailed unpaired Student's t-test was applied for analyzing differences between two groups, while a one-way ANOVA followed by Tukey's post hoc test was used for analyzing differences among $\geq 3$ groups. The association between LINC00210 expression and clinical factors in patients with glioma was analyzed using a $\chi^{2}$ test. Survival analysis was performed using a Kaplan-Meier survival curve and a log-rank test. The Spearman rank correlation test was used to analyze the correlation between the expression levels of LINC00210 and miR-328 in glioma tissues. $\mathrm{P}<0.05$ was considered to indicate a statistically significant difference.

\section{Results}

Upregulation of LINC00210 is associated with glioma progression and poor prognosis. RT-qPCR was conducted to examine the expression of LINC00210 in glioma tissues and healthy brain tissues, and it was found that LINC00210 expression was significantly higher in glioma tissues compared with healthy brain tissues (Fig. 1A). Consistent with the in vivo data, the expression of LINC00210 was also significantly upregulated in human glioma cell lines, including U251, T98G, U-373MG Uppsala and U-87MG, compared with NHAs (Fig. 1B). Moreover, survival analysis results demonstrated that patients with glioma with high LINC00210 
Table I. Association between LINC00210 expression and clinicopathological characteristics of patients with glioma.

\begin{tabular}{|c|c|c|c|c|c|}
\hline Variables & Total cases $(\mathrm{n}=54)$ & Low LINC00210 $(n=30)$ & High LINC00210 $(n=24)$ & $\chi^{2}$-value & P-value \\
\hline \multicolumn{6}{|l|}{ Age, years } \\
\hline$<50$ & 28 & 16 & 12 & 0.059 & 0.808 \\
\hline$\geq 50$ & 26 & 14 & 12 & & \\
\hline \multicolumn{6}{|l|}{ Sex } \\
\hline Male & 33 & 18 & 15 & 0.035 & 0.852 \\
\hline Female & 21 & 12 & 9 & & \\
\hline \multicolumn{6}{|c|}{ WHO stage } \\
\hline I-II & 20 & 15 & 5 & 4.864 & $0.027^{\mathrm{a}}$ \\
\hline III-IV & 34 & 15 & 19 & & \\
\hline
\end{tabular}

${ }^{a} \mathrm{P}<0.05$. WHO, World Health Organization.
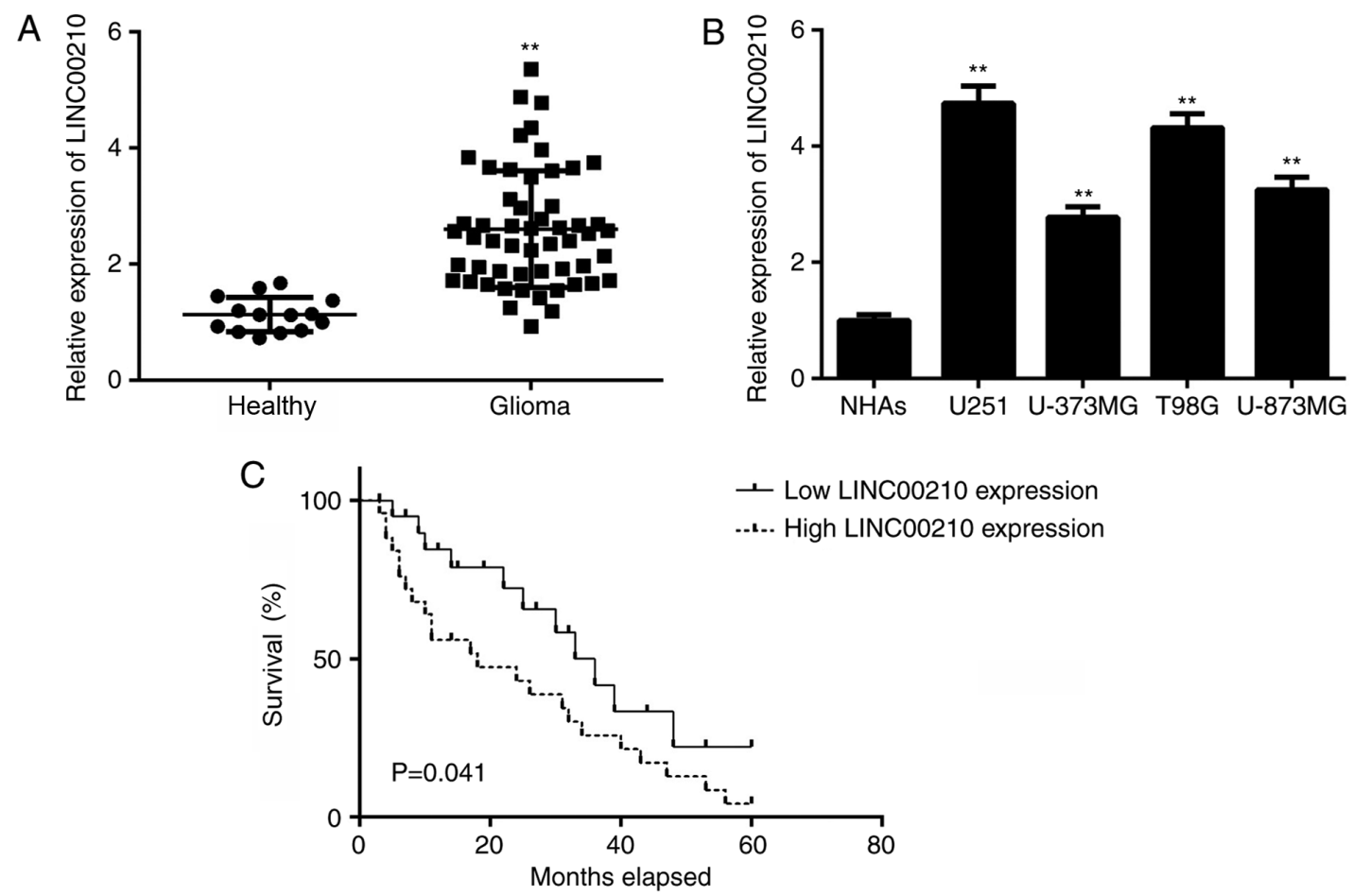

Figure 1. Upregulation of LINC00210 in glioma. (A) RT-qPCR data indicated that LINC00210 was significantly higher in glioma tissues compared with healthy brain tissues. ${ }^{* *} \mathrm{P}<0.01$ vs. healthy. (B) RT-qPCR data demonstrated that LINC00210 was significantly upregulated in glioma cell lines compared with NHAs. ${ }^{* *} \mathrm{P}<0.01$ vs. NHAs. (C) Patients with glioma with high LINC00210 expression had significantly shorter survival times compared with patients with low LINC00210 expression. NHA, normal human astrocytes; RT-qPCR, reverse transcription-quantitative PCR.

expression had significantly shorter survival times compared with patients with low LINC00210 expression (Fig. 1C). A $\chi^{2}$ test was performed to analyze the association between LINC00210 expression and clinical factors in patients with glioma. High expression of LINC00210 was positively associated with an advanced clinical stage in patients with glioma (Table I). Thus, upregulation of LINC00210 was associated with disease progression and poor prognosis in glioma.

Knockdown of LINC00210 suppresses the proliferation and migration of U251 and T98G cells. As U251 and T98G cell lines exhibited the highest expression of LINC00210 among the four glioma cell lines examined, these two cell lines were used in subsequent experiments. To further examine the role of LINC00210 in glioma cell proliferation and migration, U251 and T98G cells were transfected with NC siRNA or two LINC00210 siRNAs. After transfection, the expression levels of LINC00210 were significantly lower in the LINC00210 siRNA1 and siRNA2 groups compared with the NC siRNA group (Fig. 2A and B). The CCK-8 assay results demonstrated that the proliferation of U251 and T98G cells was significantly lower in the LINC00210 siRNA1 and siRNA2 groups compared with the NC siRNA group, indicating that knockdown of LINC00210 significantly inhibited glioma 

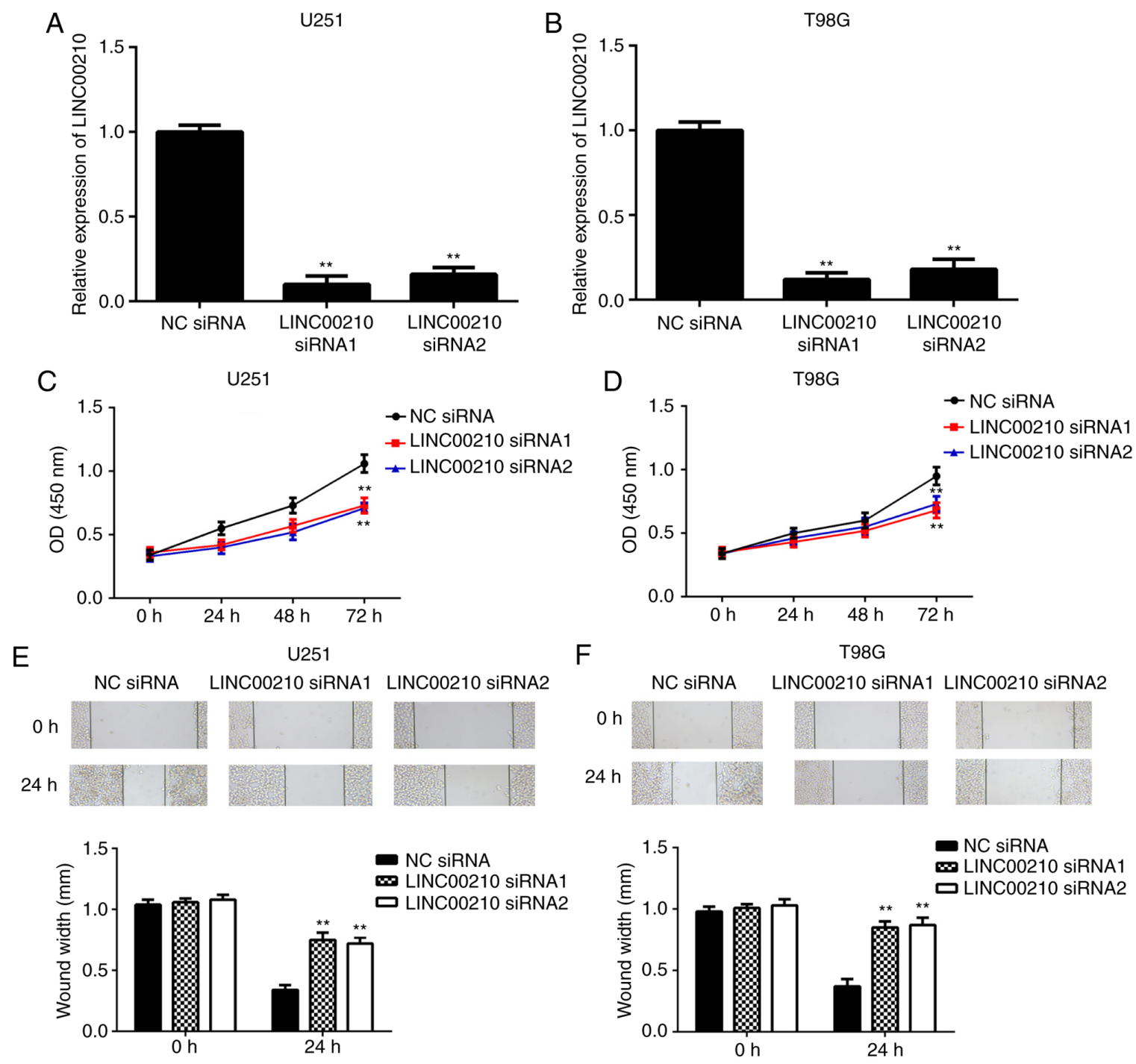

Figure 2. Knockdown of LINC00210 inhibits glioma cell proliferation and migration. U251 and T98G cells were transfected with NC siRNA or two LINC00210 siRNAs. After transfection, reverse transcription-quantitative PCR was used to examine the expression of LINC00210 in (A) U251 and (B) T98G cells. After transfection, Cell Counting Kit-8 assays were used to determine (C) U251 and (D) T98G cell proliferation. After transfection, wound healing assays were used to determine (E) U251 and (F) T98G cell migration (magnification, $\mathrm{x} 40$ ). ${ }^{* *} \mathrm{P}<0.01 \mathrm{vs.} \mathrm{NC} \mathrm{siRNA.} \mathrm{NC,} \mathrm{negative} \mathrm{control;} \mathrm{siRNA,} \mathrm{small} \mathrm{interfering} \mathrm{RNA;}$ OD, optical density.

cell proliferation (Fig. 2C and D). Similarly, the migration of glioma cells was significantly decreased after knocking down LINC00210 expression (Fig. 2E and F). These data suggested that LINC00210 may have a promoting role in glioma cell proliferation and migration.

LINC00210 directly targets miR-328 in glioma cells and tissues. It has been reported that IncRNAs can function by regulating downstream miRNAs $(13,14)$. Bioinformatics analysis predicted that miR-328 was a direct target of LINC00210, and to assess this prediction, luciferase reporter plasmids containing WT and MT LINC00210 binding sites with miR-328 were generated (Fig. 3A). U251 and T98G cells were also transfected with miR-NC or miR-328 mimic. After transfection, RT-qPCR data indicated that miR-328 expression was significantly increased in the miR-328 group compared with the miR-NC group (Fig. 3B). Luciferase reporter gene assays were then conducted in $\mathrm{U} 251$ and T98G cells. Transfection with miR-328 mimic significantly decreased the luciferase activity in the WT-LINC00210 group but did not affect the luciferase activity in the MT-LINC00210 group (Fig. 3C and D). These data indicated that LINC00210 directly targeted miR-328 in U251 and T98G cells.

It was found that the expression of miR-328 was significantly higher in U251 and T98G cells transfected with LINC00210 siRNAs compared with cells transfected with NC siRNA (Fig. 3E), suggesting that silencing LINC00210 increased the expression of miR-328 in glioma cells. To further investigate the relationship between miR-328 and LINC00210 in glioma, RT-qPCR was performed to examine the expression of miR-328 in glioma tissues. It was identified that miR-328 expression was significantly lower in glioma tissues compared with healthy brain tissues (Fig. 3F), and was also significantly downregulated in glioma cell lines (Fig. $3 \mathrm{H}$ ). In addition, the expression of miR-328 was moderately, negatively correlated with the expression of LINC00210 in glioma tissues (Fig. 3G). Thus, it was demonstrated that upregulation of LINC00210 in glioma contributed to downregulation of miR-328. 
A

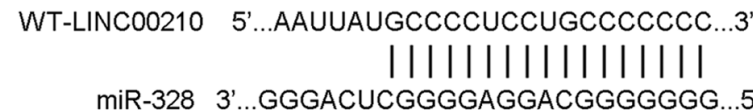

MT-LINC00210 5'...AAUUAUCGGGGAGGACGGGGGGG...3'

C

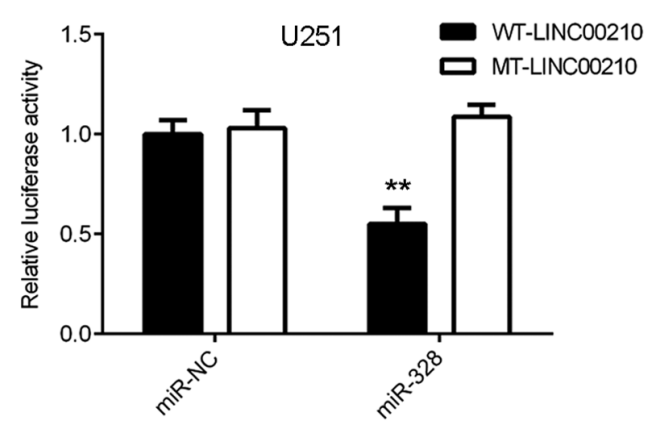

$\mathrm{E}$

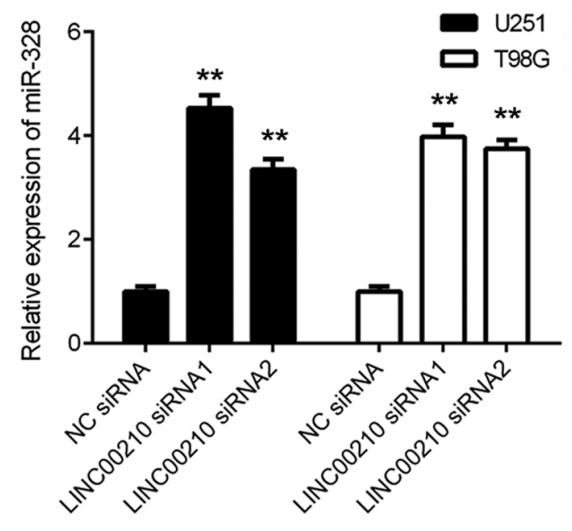

G

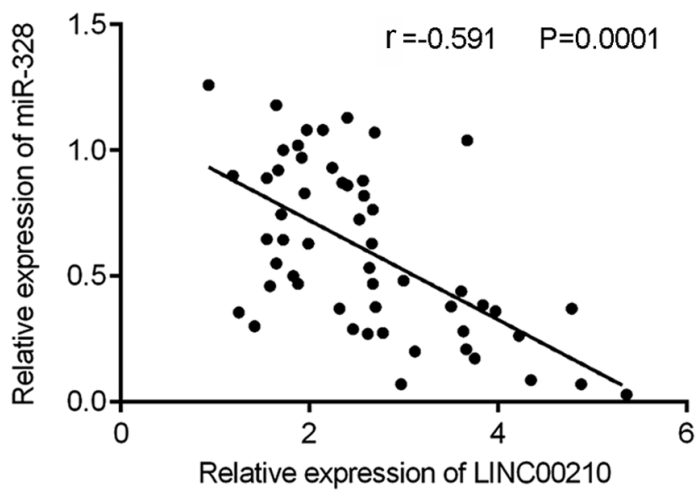

B
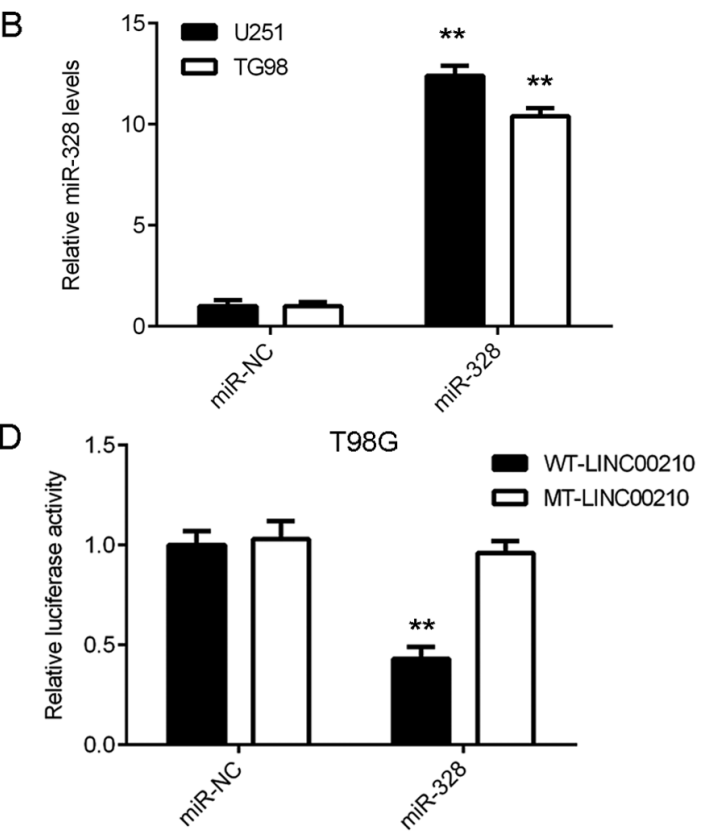

$\mathrm{F}$

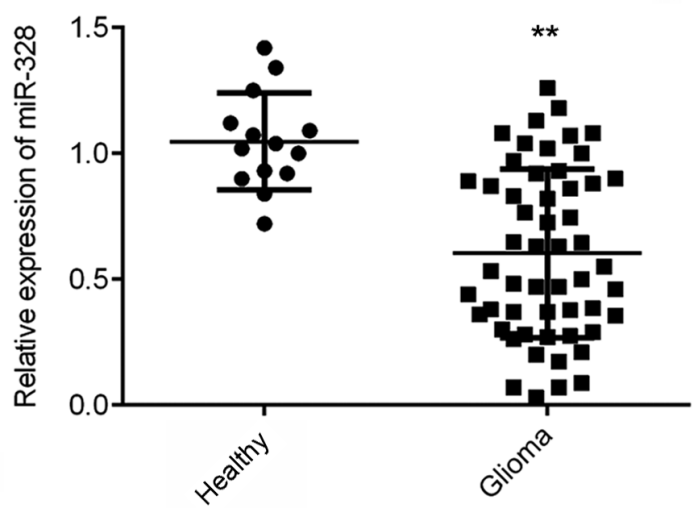

$\mathrm{H}$

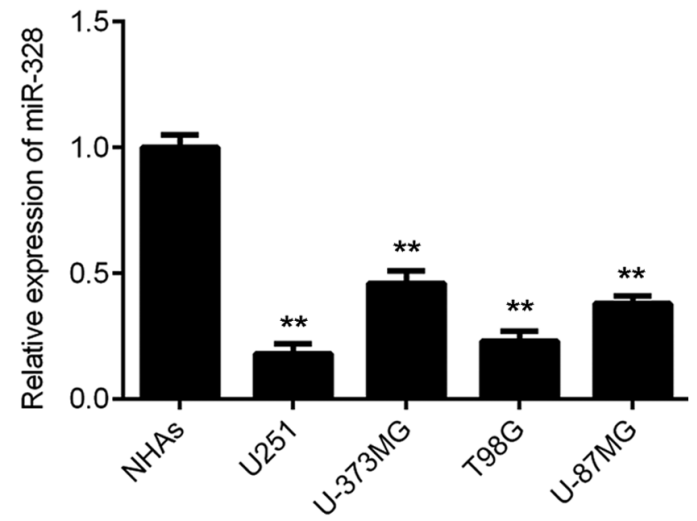

Figure 3. LINC00210 directly targets miR-328 in glioma cells and tissues. (A) WT and MT LINC00210 binding sites with miR-328. (B) U251 and T98G cells were transfected with miR-NC or miR-328 mimic. After transfection, RT-qPCR was used to examine the expression of miR-328. Luciferase reporter assay results demonstrated that transfection with the miR-328 mimic significantly decreased the luciferase activity in the WT-LINC00210 group but did not affect the luciferase activity in the MT-LINC00210 group in (C) U251 and (D) T98G cells. ** P<0.01 vs. corresponding miR-NC. (E) RT-qPCR data indicated that miR-328 expression was significantly higher in the groups transfected with the LINC00210 siRNAs compared with the NC siRNA group. ${ }^{* *} \mathrm{P}<0.01$ vs. NC siRNA. (F) RT-qPCR data identified that miR-328 expression was significantly lower in glioma tissues compared with healthy brain tissues. ${ }^{* *} \mathrm{P}<0.01$ vs. healthy. (G) Expression of miR-328 was negatively correlated with the expression of LINC00210 in glioma tissues. (H) RT-qPCR data demonstrated that miR-328 expression was significantly downregulated in glioma cell lines compared with NHAs. ${ }^{* *} \mathrm{P}<0.01$ vs. NHAs. NHA, normal human astrocytes; RT-qPCR, reverse transcription-quantitative PCR; miR, microRNA; NC, negative control; siRNA, small interfering RNA; WT, wild-type; MT, mutant type.

Knockdown of LINC00210 suppresses glioma cell proliferation and migration by increasing the expression of miR-328. Based on the aforementioned findings, it was considered that
miR-328 may be involved in LINC00210-mediated glioma cell proliferation and migration. To further test this hypothesis, U251 and T98G cells were transfected with NC inhibitor 


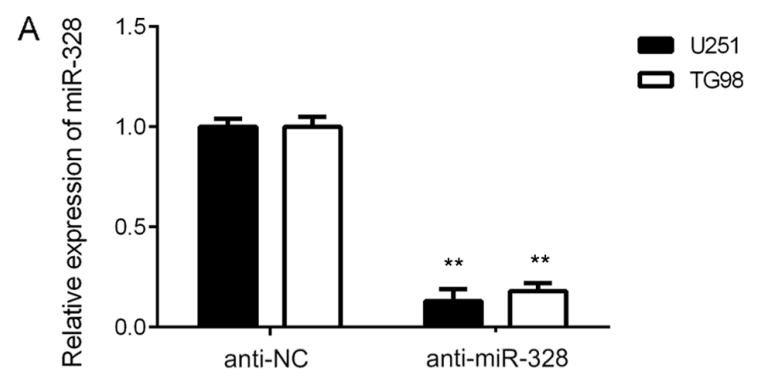

B

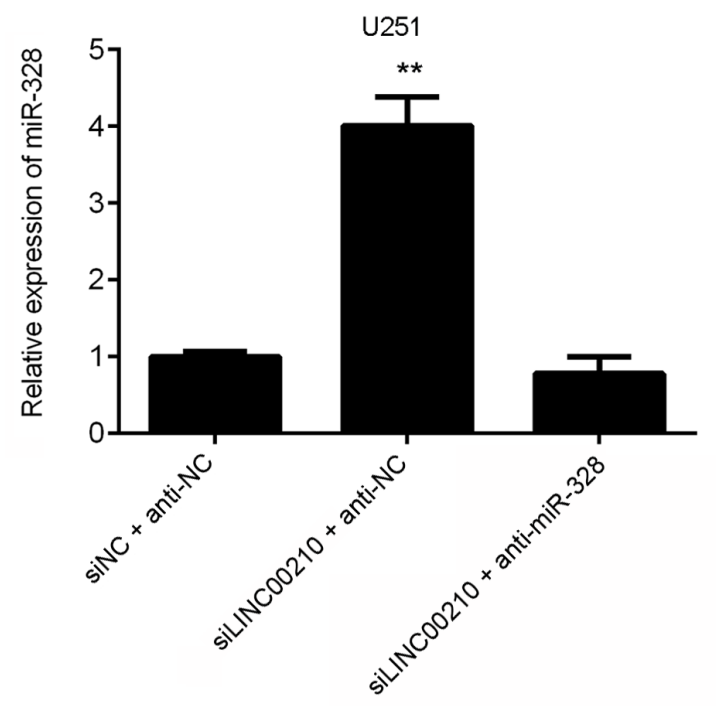

D

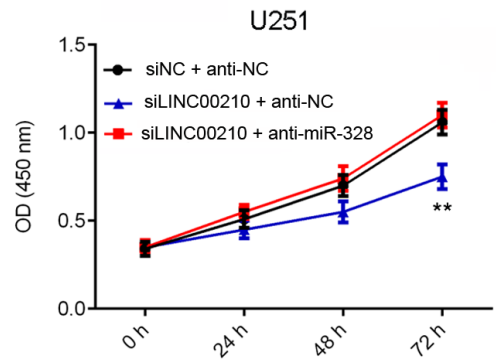

$\mathrm{F}$

$\mathrm{U} 251$
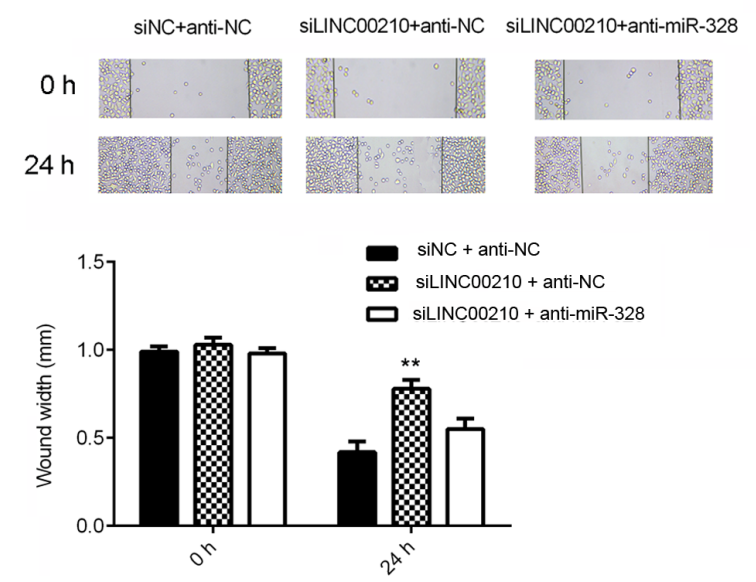

C

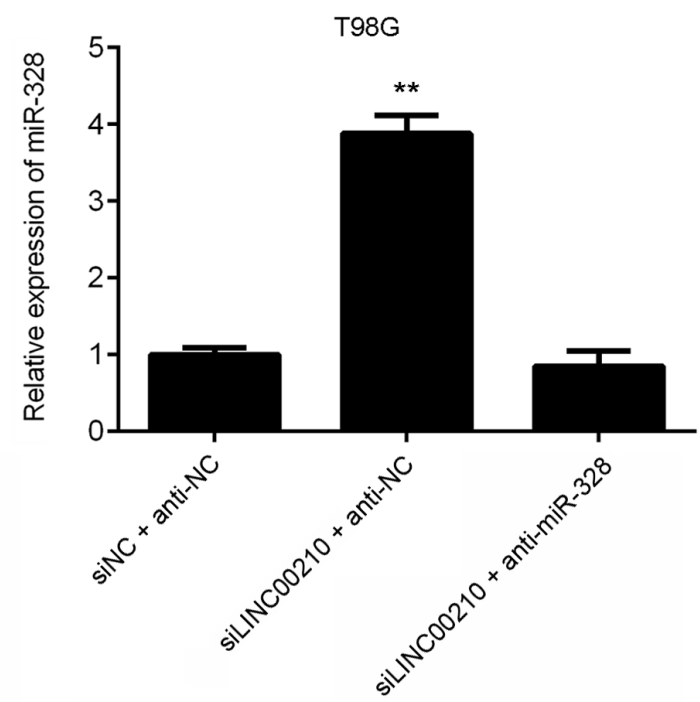

E

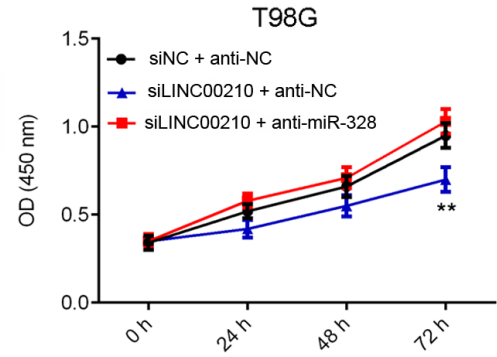

G

T98G

siNC+anti-NC siLINC00210+anti-NC siLINC00210+anti-miR-328
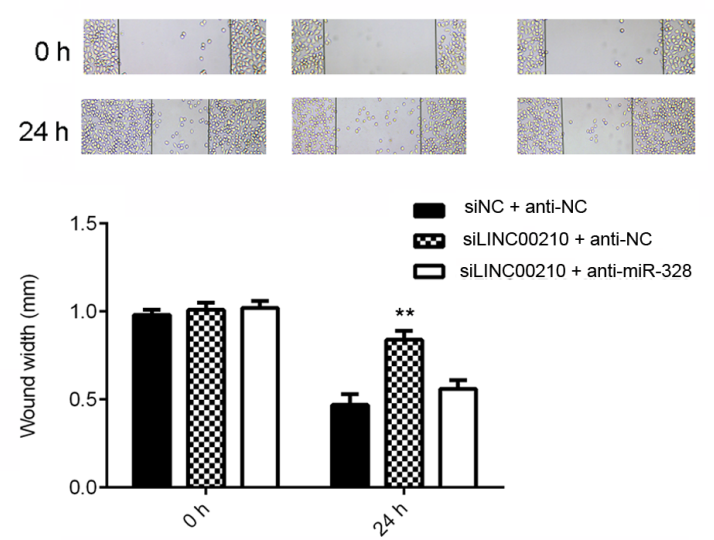

Figure 4. Knockdown of LINC00210 suppresses glioma cell proliferation and migration by increasing the expression of miR-328. (A) U251 and T98G cells were transfected with NC inhibitor or miR-328 inhibitor. After transfection, RT-qPCR was used to examine the expression of miR-328. Then, U251 and T98G cells were transfected with NC siRNA and NC inhibitor (siNC + anti-NC), LINC00210 siRNA1 and NC inhibitor (siLINC00210 + anti-NC) or LINC00210 siRNA1 and miR-328 inhibitor (siLINC00210 + anti-miR-328). After transfection, RT-qPCR was used to examine the expression of LINC00210 in (B) U251 and (C) T98G cells. After transfection, Cell Counting Kit-8 assays were used to determine (D) U251 and (E) T98G cell proliferation. After transfection, wound healing assays were used to determine (F) U251 and (G) T98G cell migration. ${ }^{* *} \mathrm{P}<0.01$ vs. siNC + anti-NC. RT-qPCR, reverse transcription-quantitative PCR; miR, microRNA; NC, negative control; siRNA, small interfering RNA; OD, optical density. 
or miR-328 inhibitor. After transfection, RT-qPCR data indicated that miR-328 expression was significantly decreased in the anti-miR-328 group compared with the anti-NC group (Fig. 4A).

As LINC00210 siRNA1 was more efficient in suppressing the expression of LINC00210 compared with siRNA2, LINC00210 siRNA1 was used in subsequent experiments. U251 and T98G cells were co-transfected with NC siRNA and $\mathrm{NC}$ inhibitor (siNC + anti-NC), LINC00210 siRNA1 and NC inhibitor (siLINC00210 + anti-NC) or LINC00210 siRNA1 and miR-328 inhibitor (siLINC00210 + anti-miR-328). After transfection, RT-qPCR results demonstrated that transfection with LINC00210 siRNA significantly increased miR-328 expression in glioma cells, which was eliminated by co-transfection with the miR-328 inhibitor (Fig. 4B and C).

CCK-8 and wound healing assays were conducted to examine cell proliferation and migration. It was found that cell proliferation was significantly inhibited in the siLINC00210 + anti-NC group when compared with that in the siNC + anti-NC group, but no significant difference in cell proliferation was observed between the siNC + anti-NC group and the siLINC00210 + miR-328 inhibitor group (Fig. 4D and E). These data indicated that silencing miR-328 eliminated the inhibitory effects on glioma cell proliferation induced by LINC00210 knockdown. Similarly, LINC00210 knockdown inhibited glioma cell migration, which was eliminated by the miR-328 inhibitor (Fig. 4F and G). Collectively, these data suggested that knockdown of LINC00210 inhibited glioma cell proliferation and migration by increasing the expression of miR-328.

\section{Discussion}

To the best of our knowledge, the expression and function of IncRNA LINC00210 in glioma had not previously been studied. The present results suggested that LINC00210 was significantly upregulated in glioma tissues, and high expression of LINC00210 was significantly associated with advanced clinical stage and poor prognosis in patients with glioma. It was identified that knockdown of LINC00210 significantly inhibited the proliferation and migration of glioma cells. Moreover, LINC00210 could directly target miR-328 in glioma cells, and miR-328 expression was negatively correlated with LINC00210 expression in glioma tissues. Knocking down LINC00210 significantly promoted the expression of miR-328 in glioma cells. Furthermore, silencing miR-328 impaired the inhibitory effects of LINC00210 knockdown on the proliferation and migration of U251 and T98G cells.

It has been well-established that lncRNAs participate in regulating physiological and pathological processes, such as tumor development and progression $(6,7)$. Numerous lncRNAs have been reported to exert important roles in glioma. For instance, the lncRNA nuclear paraspeckle assembly transcript 1 (NEAT1) is upregulated in glioma, and NEAT1 inhibition reduces glioma cell viability, migration and invasion, indicating that NEAT exerts an oncogenic role in glioma (5).

LINC00210 is a newly discovered lncRNA and has rarely been studied. Fu et al (17) revealed that LINC00210 was frequently upregulated in liver cancer tissues and liver tumor-initiating cells (TICs). These authors also observed that
LINC00210 promoted the self-renewal and tumor initiating capacity of liver TICs by interacting with $\beta$-catenin interacting protein 1 and activating Wnt/ $\beta$-catenin signaling (17). However, to the best of our knowledge, the expression pattern and function of LINC00210 in glioma have not been previously reported. The present study revealed for the first time that the expression of LINC00210 was significantly higher in glioma tissues and cell lines compared with healthy brain tissues and NHAs. Moreover, it was identified that high LINC00210 expression was associated with advanced clinical stage and poor prognosis in patients, suggesting that upregulation of LINC00210 may promote glioma progression. It was also demonstrated that knocking down LINC00210 expression via siRNA significantly inhibited glioma cell proliferation and migration, indicating that targeting LINC00210 may be a promising therapeutic strategy for glioma treatment in the future.

miRNAs, a class of small non-coding RNAs, have been reported to function as key regulators of gene expression via binding to the 3'-untranslated region of their target mRNAs and causing translation suppression or RNA degradation $(13,19)$. Various miRNAs also possess promoting or suppressing roles in different human cancer types, including glioma $(10,20,21)$. For instance, miR-365 inhibits proliferation, migration and invasion of glioma by targeting phosphoinositide-3-kinase regulatory subunit 3 (22), while miR-25 promotes glioma cell proliferation by targeting cyclin dependent kinase inhibitor $1 \mathrm{C}$ (23).

Previous studies have reported that miR-328 acts as a tumor suppressor in glioma $(24,25)$. For example, Wu et al (24) revealed that miR-328 expression was decreased in high-grade glioma and was associated with worse survival in primary glioblastoma. The present results also indicated that the expression of miR-328 was lower in glioma tissues compared with healthy brain tissues. In addition, Yuan et al (25) observed that miR-328 was a favorable prognostic marker in glioma as it suppressed the invasive and proliferative phenotypes of glioma cells. Wang et al (26) also reported that enhancer of zeste 2 polycomb repressive complex 2 subunit could promote $\beta$-catenin signaling by inhibiting miR-328 expression, which further promoted glioma cell proliferation and glucose metabolism. The present study demonstrated that miR-328 was a target of LINC00210 in glioma cells, and found a negative correlation between the expression levels of LINC00210 and miR-328. These findings suggested that the lower expression of miR-328 may be due to the upregulation of LINC00210 in glioma. Moreover, it was identified that silencing miR-328 eliminated the inhibitory effects on glioma cell proliferation induced by LINC00210 knockdown, suggesting that LINC00210 serves a promoting role in glioma cell proliferation and migration by inhibiting miR-328. Similarly, Zhang et al (27) reported that LINC00210 promoted nasopharyngeal carcinoma tumorigenesis by regulating miR-328-5p expression and activating the NOTCH3 pathway. The LINC00210/miR-328 axis may also serve important roles in cancer types other than glioma and nasopharyngeal cancer, which should be further studied in the future. However, the limitations of the present study are that the findings were not confirmed in vivo in animal models.

In conclusion, the present study demonstrated that upregulation of LINC00210 promoted glioma progression and predicted poor prognosis. Furthermore, it was suggested that 
LINC00210 exerted an oncogenic role in glioma cell proliferation and migration via regulating miR-328 expression. The results of the present study may facilitate the development of glioma treatment.

\section{Acknowledgements}

Not applicable.

\section{Funding}

This study was supported by the Surface Project of Hunan Natural Science Foundation (grant no. 2018JJ2607).

\section{Availability of data and materials}

The datasets used and/or analyzed during the current study are available from the corresponding author on reasonable request.

\section{Authors' contributions}

ZW designed this study. HW collected clinical samples and performed analysis of clinical data. HY performed statistical analysis. TC, JD and QL performed experiments. ZW and QL wrote the manuscript. All authors read and approved the final manuscript.

\section{Ethics approval and consent to participate}

This study was approved by the Ethics Committee of Third Xiangya Hospital of Central South University (Hunan, China). Written informed consents were obtained from patients.

\section{Patient consent for publication}

Not applicable.

\section{Competing interests}

The authors declare that they have no competing interests.

\section{References}

1. Siegel RL, Miller KD and Jemal A: Cancer Statistics, 2017. CA Cancer J Clin 67: 7-30, 2017.

2. Siegel RL, Miller KD and Jemal A: Cancer statistics, 2015. CA Cancer J Clin 65: 5-29, 2015.

3. Peng $\mathrm{Z}$, Liu $\mathrm{C}$ and $\mathrm{Wu} \mathrm{M}$ : New insights into long noncoding RNAs and their roles in glioma. Mol Cancer 17: 61, 2018.

4. Anjum K, Shagufta BI, Abbas SQ, Patel S, Khan I, Shah SAA, Akhter $\mathrm{N}$ and Hassan SSU: Current status and future therapeutic perspectives of glioblastoma multiforme (GBM) therapy: A review. Biomed Pharmacother 92: 681-689, 2017.

5. Zhou K, Zhang C, Yao H, Zhang X, Zhou Y, Che Y and Huang Y: Knockdown of long non-coding RNA NEAT1 inhibits glioma cell migration and invasion via modulation of SOX 2 targeted by miR-132. Mol Cancer 17: 105, 2018.

6. Smolle MA and Pichler M: The role of long non-coding RNAs in osteosarcoma. Noncoding RNA 4: 7, 2018.

7. Xu S, Kong D, Chen Q, Ping Y and Pang D: Oncogenic long noncoding RNA landscape in breast cancer. Mol Cancer 16: 129, 2017.
8. Zhang CJ, Liu C, Wang YX, Zhu N, Hu ZY, Liao DF and Qin L: Long non-coding RNA-SRA promotes neointimal hyperplasia and vascular smooth muscle cells proliferation via MEK-ERK-CREB pathway. Vascul Pharmacol 116: 16-23, 2019.

9. He D, Zeng H, Chen J, Xiao L, Zhao Y and Liu N: H19 regulates trophoblastic spheroid adhesion by competitively binding to let-7. Reproduction 157: 423-430, 2019.

10. Ma Z: Downregulation of SETD8 by miR-382 is involved in glioma progression. Pathol Res Pract 214: 356-360, 2018.

11. Bo H, Fan L, Gong Z, Liu Z, Shi L, Guo C, Li X, Liao Q, Zhang W, Zhou M, et al: Upregulation and hypomethylation of lncRNA AFAP1AS1 predicts a poor prognosis and promotes the migration and invasion of cervical cancer. Oncol Rep 41: 2431-2439, 2019.

12. Jiang H, Xiong W, Chen L, Lv Z, Yang C and Li Y: Knockdown of the long noncoding RNA HOTTIP inhibits cell proliferation and enhances cell sensitivity to cisplatin by suppressing the Wnt $/ \beta$-catenin pathway in prostate cancer. J Cell Biochem 120: 8965-8974, 2019.

13. Ambros V: The functions of animal microRNAs. Nature 431: 350-355, 2004

14. Zhou S, Yu L, Xiong M and Dai G: 1ncRNA SNHG12 promotes tumorigenesis and metastasis in osteosarcoma by upregulating Notch2 by sponging miR-195-5p. Biochem Biophys Res Commun 495: 1822-1832, 2018.

15. Tan P, Guo YH, Zhan JK, Long LM, Xu ML, Ye L, Ma XY, Cui XJ and Wang HQ: IncRNA-ANRIL inhibits cell senescence of vascular smooth muscle cells by regulating miR-181a/Sirt1. Biochem Cell Biol 97: 571-580, 2019.

16. Lin C, Zhang Y, Chen Y and Bai Y: Long noncoding RNA LINC01234 promotes serine hydroxymethyltransferase 2 expression and proliferation by competitively binding miR-642a-5p in colon cancer. Cell Death Dis 10: 137, 2019.

17. Fu X, Zhu X, Qin F, Zhang Y, Lin J, Ding Y, Yang Z, Shang Y, Wang L, Zhang Q and Gao Q: Linc00210 drives Wnt/ $\beta$-catenin signaling activation and liver tumor progression through CTNNBIP1-dependent manner. Mol Cancer 17: 73, 2018.

18. Livak KJ and Schmittgen TD: Analysis of relative gene expression data using real-time quantitative PCR and the 2(-Delta Delta C(T)) method. Methods 25: 402-408, 2001.

19. Bartel DP: MicroRNAs: Genomics, biogenesis, mechanism, and function. Cell 116: 281-297, 2004.

20. Tan Z, Zhao J and Jiang Y: miR-634 sensitizes glioma cells to temozolomide by targeting CYR61 through Raf-ERK signaling pathway. Cancer Med 7: 913-921, 2018.

21. Xiao F, Li Y, Wan Y and Xue M: MircroRNA-139 sensitizes ovarian cancer cell to cisplatin-based chemotherapy through regulation of ATP7A/B. Cancer Chemother Pharmacol 81: 935-947, 2018.

22. Zhu Y, Zhao H, Rao M and Xu S: MicroRNA-365 inhibits proliferation, migration and invasion of glioma by targeting PIK3R3. Oncol Rep 37: 2185-2192, 2017.

23. Zhang J, Gong X, Tian K, Chen D, Sun J, Wang G and Guo M: miR-25 promotes glioma cell proliferation by targeting CDKN1C. Biomed Pharmacother 71: 7-14, 2015.

24. Wu Z, Sun L, Wang H, Yao J, Jiang C, Xu W and Yang Z: miR-328 expression is decreased in high-grade gliomas and is associated with worse survival in primary glioblastoma. PLoS One 7: e47270, 2012.

25. Yuan J, Zheng Z, Zheng Y, Lu X, Xu L and Lin L: microRNA-328 is a favorable prognostic marker in human glioma via suppressing invasive and proliferative phenotypes of malignant cells. Int $\mathbf{J}$ Neurosci 126: 145-153, 2016.

26. Wang Y, Wang M, Wei W, Han D, Chen X, Hu Q, Yu T, Liu N, You Y and Zhang J: Disruption of the EZH2/miRNA/ $\beta$-catenin signaling suppresses aerobic glycolysis in glioma. Oncotarget 7: 49450-49458, 2016.

27. Zhang S, Li P, Zhao L and Xu L: LINC00210 as a miR-328-5p sponge promotes nasopharyngeal carcinoma tumorigenesis by activating NOTCH3 pathway. Biosci Rep 38: BSR20181168, 2018.

(i) $($ ) This work is licensed under a Creative Commons Attribution-NonCommercial-NoDerivatives 4.0 International (CC BY-NC-ND 4.0) License. 